\title{
IBL - ATLAS Pixel Upgrade
}

\section{Tobias Flick*i}

University of Wuppertal

E-mail: tobias.flickecern.ch

The upgrade of the ATLAS Detector will progress through different phases as we prepare for the transition to the sLHC. The first upgrade for the innermost tracker, the Pixel Detector, will be the IBL, an additional insertable B-Layer. This layer will be included into the Pixel Detector to compensate for the degradation of the B-layer, the one currently closest to the beam pipe. The IBL project consists of many R\&D projects covering electronics, sensors, mechanics, readout, etc. It will be the first step of the upgrade and will address already some of the aspects to be covered for a sLHC tracker detector. In this paper we discuss the different aspects of developing a more radiation hard Pixel Detector layer which will be placed closer to the interaction point, and we provide an overview of the status of the project.

VERTEX 2009 (18th workshop) - VERTEX 2009

September 13 - 182009

Veluwe, the Netherlands

\footnotetext{
* Speaker.

${ }^{\dagger}$ for the IBL developing group.
} 


\section{Introduction}

The ATLAS [1] Pixel Detector [2] (see Fig. 1) is the innermost sub-detector of ATLAS and therefore the one suffering the most from the severe radiation environment induced by the LHC in ATLAS. Due to the proximity of the B-layer sensors (the layer closest to the beam pipe) to the interaction point, an upgrade of the whole innermost layer is necessary after about 5 years of operation, which coincides with the timing of the LHC phase-I upgrade. Given the anticipated luminosity profile and the installation foreseen in 2014 the current B-layer will still participate in the data taking, but its efficiency will decrease with further time. Taking into account the time needed to replace the existing B-Layer would be more than a year due to the activation of the material which needs to be deinstalled the only possible solution to provide an additional space point close to the interaction point is to introduce an additional layer to the existing detector. The new insertable B-Layer (IBL) [3] is currently under development and all items which are addressed here are still preliminary. The general idea of how to realize the new layer will be described here, since for some items several options need still to be explored while for others the details will certainly change in the course of the development process.

The new layer should be realized using new technologies; chips developed using current technology, and updated off-detector electronics using modern components. It must withstand the irradiation until the end of LHC. A study for the $1 \mathrm{MeV}$ equivalent fluence for different radii is given in Fig. 2.

The IBL needs to be integrated as a part of the existing Pixel Detector. Therefore it will be the 4th Pixel layer and must be compatible with the control system and operation of the existing detector. Since almost all of the addressed items are still under development, this paper outlines the most important components and describes the basic idea for the development.

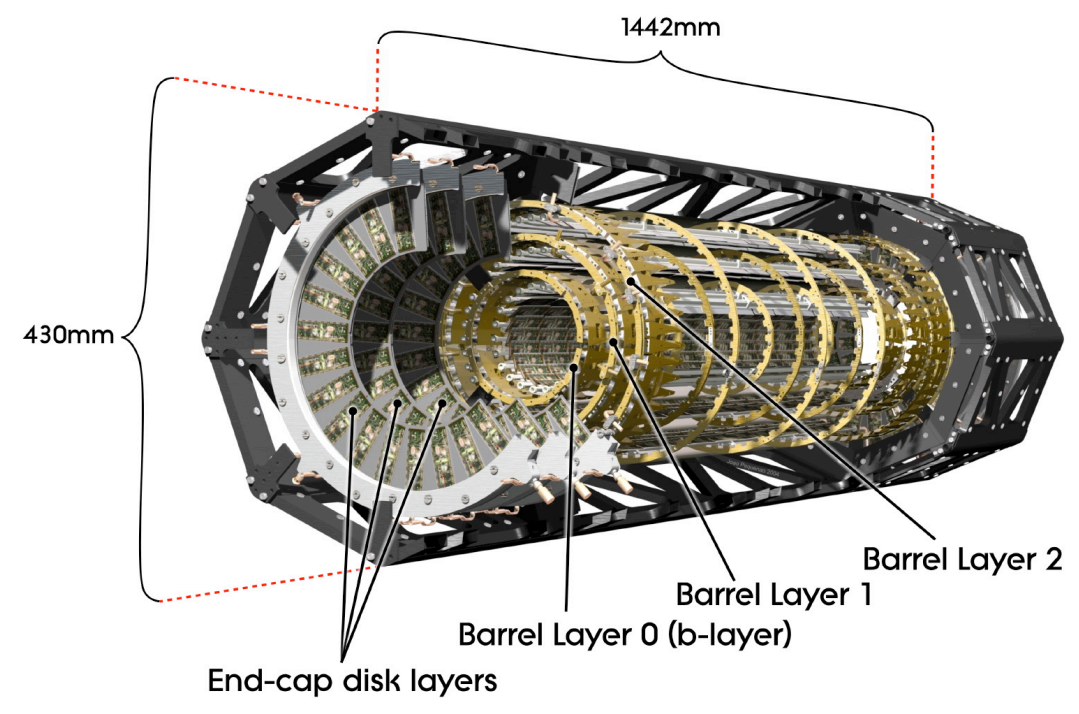

Figure 1: The current ATLAS Pixel Detector 


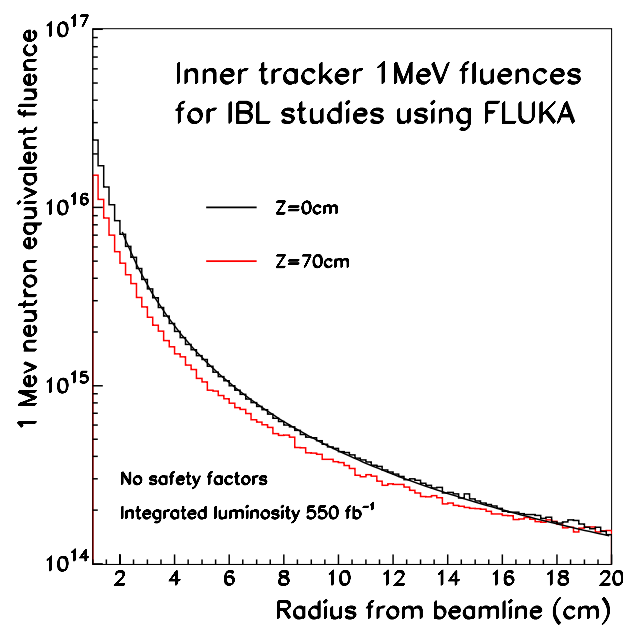

Figure 2: $1 \mathrm{MeV}$ fluences versus radius $\mathrm{R}$ from the beam line, for the cases $\mathrm{z}=0 \mathrm{~cm}$ and $\mathrm{z}=70 \mathrm{~cm}$ [4]. The parameterization is $\Phi(r)=\left(\frac{2.9}{r^{2}}+\frac{0.14}{r}\right) \cdot 10^{16}$

\section{Layout}

The baseline for the geometry of the IBL is a barrel layer consisting of 14 staves. Each of these will have 16 modules, which each consist of two front-end chips bonded to a sensor. This can be either two independent sensor tiles or one common sensor tile (see later). The staves are tilted by $14^{\circ}$ to guarantee an overlap region in $\Phi$. The inner radius of the IBL will be $\sim 32 \mathrm{~mm}$, which can only be realized with a beam pipe inner radius of $25 \mathrm{~mm}$. The outer radius of the IBL will be $\sim 38.3 \mathrm{~mm}$, which leaves enough clearance to the current innermost Pixel Detector layer. A section of the baseline design is sketched in Fig. 3. The sensor surface of the IBL will only $\sim 0.2 \mathrm{~m}^{2}$ and located at a radius of $\sim 33 \mathrm{~mm}$ from the interaction point. The dimensions for the layout of the IBL are summarized in Table 1 . The total material budget sums up to a radiation length of $X / X_{0} \approx 1.5 \%$.

\begin{tabular}{|l|c|}
\hline Staves & 14 \\
\hline Length of stave & $80 \mathrm{~cm}$ \\
\hline Width of stave & $2 \mathrm{~cm}$ \\
\hline Inner radius & $3.2 \mathrm{~cm}$ \\
\hline Outer radius & $3.835 \mathrm{~cm}$ \\
\hline Tilt angle & $14^{\circ}$ \\
\hline
\end{tabular}

Table 1: Planned dimensions (current status).

\section{Electronics}

The front-end chip which is foreseen for the IBL is the FE-I4 chip [5] [6]. It serves 26880 
pixels arranged in 80 columns ( $250 \mu \mathrm{m}$ pitch) by 336 rows $(50 \mu \mathrm{m}$ pitch). It is designed in a $130 \mathrm{~nm}$ feature size bulk CMOS process. The chips are connected to the sensors via bump bonding using DC coupling and collecting negative charges.

Each FE-I4 pixel contains an independently running amplification stage with adjustable signal shaping, followed by a discriminator with an independently adjustable threshold. The chip keeps track of the threshold crossing time of each discriminator as well as the time over threshold (TOT) with 4-bit resolution, in counts of an externally supplied clock, nominally $40 \mathrm{MHz}$. Information from all hits triggering the discriminator is kept in the chip for a latency interval, programmable up to 256 cycles of the external clock. Within this latency interval, the information can be retrieved by supplying an external trigger. The data output is transmitted on a serial line using a currentbalanced pair (similar to LVDS). The primary output mode is $8 \mathrm{~b} / 10 \mathrm{~b}$ encoded with $160 \mathrm{Mb} / \mathrm{s}$ rate. The FE-I4 is controlled by a serial LVDS input synchronized to the external clock. No additional $\mathrm{I} / \mathrm{O}$ connections are required for regular operation, but several others are provided for testing. The basic specifications of the FE-I4 are listed in Table 2. The first iteration of chips will provide capabilities to test for different powering options. A DC-DC converter is integrated as well as serial powering regulators. With the first submission of chips expected to be received back in 2010, testing of these options will help to choose the powering scheme for the on-detector components.

\section{Sensors}

The functional module outline for the IBL can be satisfied with either single chip assemblies that have very narrow sensor edges (Fig. 4(a)), or two-chip assemblies with slightly larger edges of insensitive material (Fig. 4(b)). Since there is no shingling in the $\mathrm{Z}$ direction of the IBL modules, due to the extreme radial space constraints, these options result in slightly different active coverage. For single-chip (two-chip) assemblies the nominal active coverage for particles normal to the beam

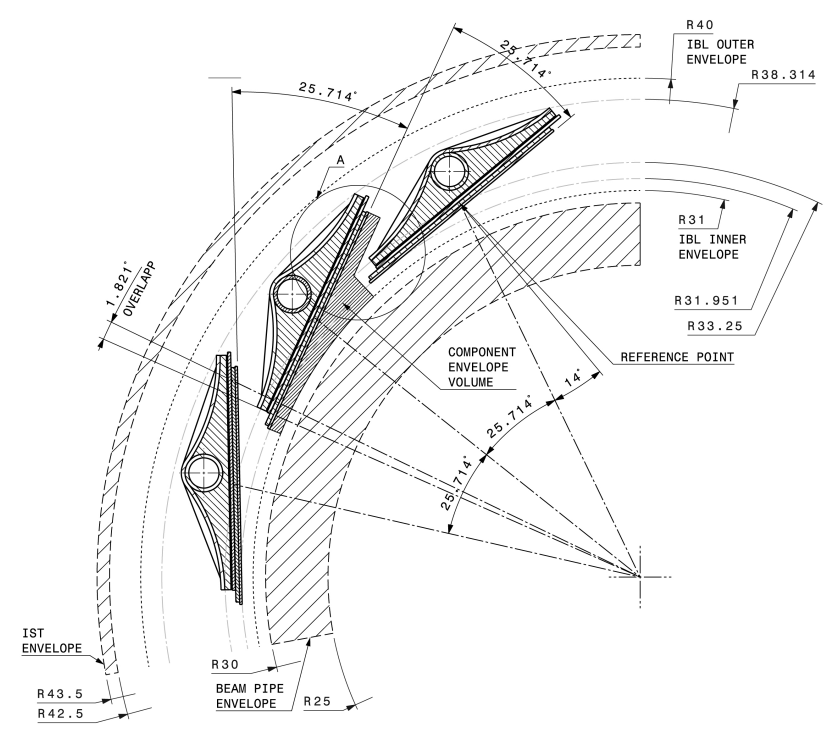

Figure 3: Radial dimensions in the IBL baseline layout. 


\begin{tabular}{|l|c|c|}
\hline \multicolumn{1}{|c|}{ Item } & Value & Units \\
\hline Pixel size & $50 \times 250$ & $\mu \mathrm{m}^{2}$ \\
\hline Maximum charge & 100,000 & $\mathrm{e}^{-}$ \\
\hline Pixel array size & $80 \times 336$ & Col $\times$ Row \\
\hline Hit-trigger association resolution & 25 & $\mathrm{~ns}$ \\
\hline Same pixel two-hit discrimination (time) & 400 & $\mathrm{~ns}$ \\
\hline Single channel ENC sigma (400 fF) & $<300$ & $\mathrm{e}^{-}$ \\
\hline Tuned threshold dispersion & $<100$ & $\mathrm{e}^{-}$ \\
\hline ADC method & Time Over Threshold (TOT) & \\
\hline Radiation tolerance (specs met at this dose) & 250 & $\mathrm{MRad}$ \\
\hline Average hit rate with $<1 \%$ data loss & 400 & $\mathrm{MHz} / \mathrm{cm}^{2}$ \\
\hline Nominal operation voltage for main chip & $1.2-1.5$ & $\mathrm{~V}$ \\
\hline Nominal currrent for main chip & $\sim 0.6$ & $\mathrm{~A}$ \\
\hline
\end{tabular}

Table 2: Basic specifications for FE-I4.

is $98.8 \%(97.4 \%)$ of the full solid angle. A gap of $100 \mu \mathrm{m}(200 \mu \mathrm{m})$ between single-chip (twochip) assemblies has been assumed in order to take into account the need for higher bias voltages in case of the 2-chip (planar) sensors. A $200 \mu \mathrm{m}$ gap is considered sufficient for adding a polyimide insulation film, if necessary.

There are three candidate sensor technologies (planar silicon, diamond, 3D silicon) that may meet the IBL requirements with their respective trade-offs. The module format required for the IBL can be satisfied with any of these technologies, thus permitting the overall design to be somewhat independent. However, the two parameters operating temperature and bias voltage are different for each technology and have serious implications on the rest of the system.

Planar sensors require the lowest temperature and highest bias voltage within the three sensor options, but have very well understood manufacturing sources, mechanical properties, are available at relatively low cost, and offer a high yield.

The 3-D sensors require the lowest bias voltage, an intermediate operating temperature, and achieve the highest geometrical acceptance due to their active edges, but their manufacturability with high yield and good uniformity must be demonstrated.

Diamond sensors require the least cooling and have similar bias voltage requirements as planar sensors, but their manufacturability with high yield, at moderate cost, and with good uniformity have also still to be demonstrated.

For all three sensor types the charge collection with increasing dose has to be quantified as a function of operational conditions. While the charge collection efficiency does not directly depend on temperature, the power and leakage current do which in turn affects the charge collection efficiency. All these studies are under investigation for the different kinds of sensors and the specific description of the sensor types can be found in Refs. [7], [8], and [9].

Charge collection is not a decisive figure of merit by itself. A significant factor in choosing a sensor technology will be the electrical performance with the FE-I4 chip. In particular, the minimum stable operating threshold is considered to be the decisive parameter that determines 


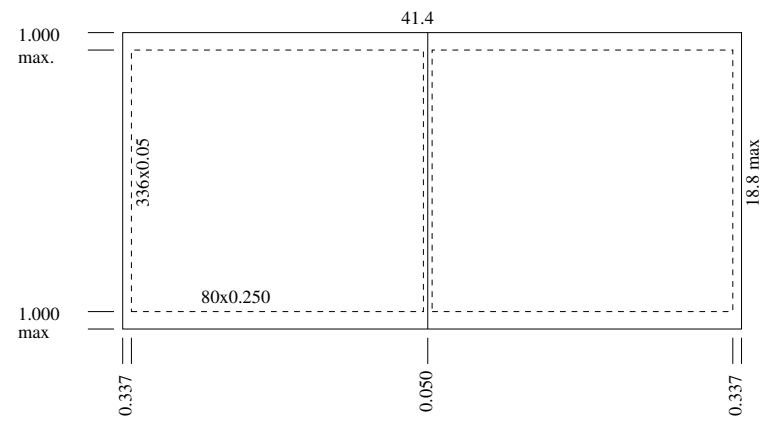

(a) IBL Envelope for 2 single-chip 3-D modules. All numbers are given in $\mathrm{mm}$.

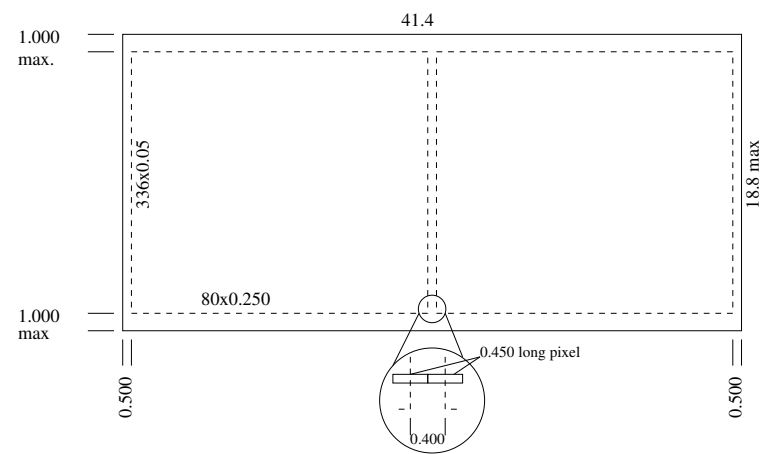

(b) IBL 2-chip planar sensor tile module. All numbers are given in $\mathrm{mm}$.

Figure 4: Module format layouts as been foreseen for different sensor types.

the required charge at the sensor's end of life, and not the single pixel white noise. However, the minimum stable threshold can not be determined with confidence from simulations, because it depends on coherent effects within the chip and sensor assembly. An analog simulation of the full system has not been technically feasible, and the exact impedance network presented by an irradiated, bump bonded sensor is not well known. Measurements on bump-bonded assemblies will provide critical input to a sensor technology choice once the FE-I4 chip is available.

\section{Mechanics}

The mechanical structure which is under development for the IBL is made from a carboncarbon carrier filled with carbon foam and containing a cooling pipe. Several design options are under investigation. The mechanical properties of stiffness, stability under thermal stress, and low mass must be optimized in conjunction with the thermal behavior, which is important for controlling the temperature of the modules (sensor and electronics). The cooling fluid choice will be made between $\mathrm{C}_{3} \mathrm{~F}_{8}$, as used in the current silicon detectors in ATLAS and therefore well known in handling and behavior, and $\mathrm{CO}_{2}$ which will be an option for upgraded detectors and is already used in LHCb. Different layout options are under investigation. Prototypes of different solutions have been built or are under construction to perform measurements for comparison with simulation 


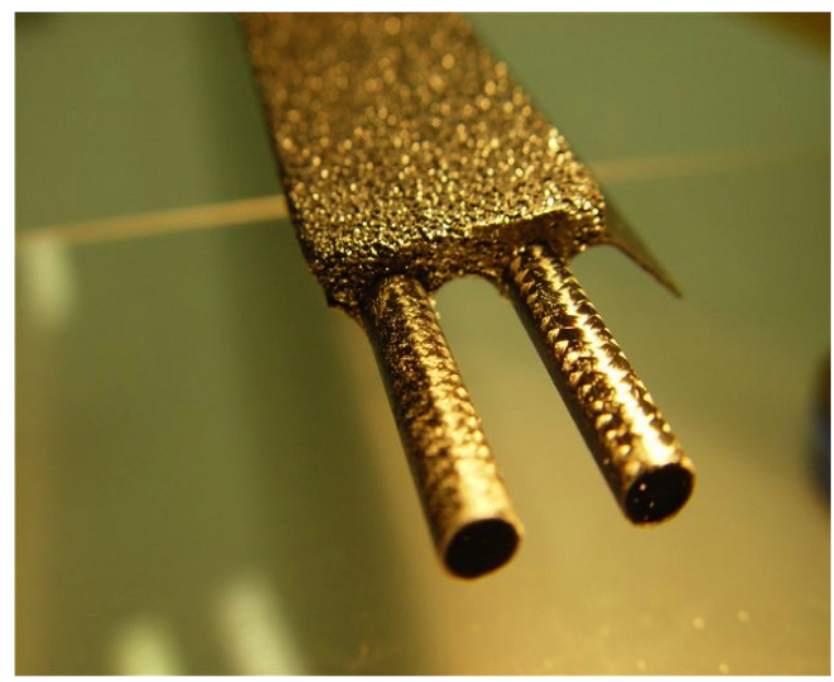

Figure 5: Prototype of a full carbon fibre homogeneous stave. Two carbon fibre pipes, which are fully qualified for the IBL usage have been integrated into the carbon foam.

results. Heat load and cooling measurements, mechanical stress tests, etc., are to be performed. Options in the design phase are single and double pipe solutions, usage of light and heavy foam, pipe material choices between carbon fibre and titanium. Fig. 5 shows the prototype of the full carbon stave equipped with two $3 \mathrm{~mm}$ inner diameter carbon fibre pipes.

\section{Readout}

The IBL will be operated as part of the current Pixel Detector and therefore the detector steering and control as well as the readout must be compatible with the present detectors. The communication with the IBL will be performed via an optical transmission line which is similar to that currently used by the Pixel Detector. The down link to the detector will be operated at $40 \mathrm{Mb} / \mathrm{s}$. Clock and commands are encoded into one bi-phase-mark-encoded stream which is sent to the detector. The clock and data will be decoded on-detector on the optoboard, which serves as the optical-electrical interface. The clock and the data signals per module are transferred via electrical lines to the detector modules. The output data is foreseen to be transmitted at $160 \mathrm{Mb} / \mathrm{s}$. The front-end chip sends $8 \mathrm{~b} / 10 \mathrm{~b}$ encoded data via an electrical line to the optoboard from where it is transmitted via optical fibres to the off-detector electronics.

In the counting room the electronics need to be upgraded as well, as the readout speed and scheme is different from the present system. The two card types, the Read Out Driver (ROD) and the Back of Crate card (BOC), are paired together in VME crates. The ROD serves as control machine and the BOC as optical interface. The cards are being designed to maintain compatibility with the existing readout electronics, with the additional benefit that the IBL cards may also be used with the present detector, either as spares or to increase the readout bandwidth.

\section{Summary}

The IBL project was initiated in July 2009 and combines many projects aimed at developing a 
$4^{\text {th }}$ ATLAS Pixel Detector layer to be installed in 2014. The mechanics will be realized in carboncarbon and carbon-foam structures (staves) to be set up in a turbine structure with 14 staves around the beam pipe.

The sensor will be chosen from three candidates (planar silicon, 3D silicon, diamond) and will be connected to front-end chips which are currently in the prototype phase (FE-I4). The assembled sensor and chip package will undergo a complete testing and qualification cycle during the decision process.

The on-detector electronics is read out via an optical link terminating in the counting rooms. The off-detector readout hardware needs a new design and development in order to accommodate the higher bandwidth and the different transmission protocols for the IBL.

These recently developed components need to be integrated into the existing ATLAS Pixel Detector as a $4^{\text {th }}$ layer. It will be operated as part of the Pixel Detector and therefore the software for data acquisition and detector control is constrained to fit into the existing software environment.

The scheduled installation date is end of 2014. By then all the components need to be produced and tested individually as well as in a combined system test.

\section{Acknowledgements}

Acknowledgements are extended to all colleagues in the IBL projects who are working on the different parts of this new layer for the Pixel Detector. Thanks be to all of you who provided input to the talk and to this paper.

\section{References}

[1] G. Aad, et al., "The ATLAS experiment at the Large Hadron Collider," JINST 3, S08003 (2008).

[2] G. Aad et al., "ATLAS Pixel Detector electronics and sensors," JINST 3, P07007 (2008).

[3] Clark, A. and Mornacchi, G., "ATLAS B-Layer Task Force final report," CERN, ATU-SYS-MR-0001, 2009

[4] I. Dawson, "IBL irradiation studies", to be published, currently to be found at https://twiki.cern.ch/twiki/bin/view/Atlas/RadiationBackgroundSimulations\#IBL_studies_Under_Construction

[5] M. Barbero et al., "A new ATLAS pixel front-end IC for upgraded LHC luminosity," Nucl. Instrum. Meth. A 604 (2009) 397.

[6] M. Garcia-Sciveres et al., "The FE-I4 Pixel Readout Integrated Circuit," to appear in Nucl. Instr. \& Meth. A

[7] A. Machiolo et al., "RD50 Recent Results - Development of radiation hard sensors for SLHC," Conference contribution to VERTEX 2009, September 13-19, 2009, Veluwe, The Netherlands

[8] C. Via et al., "3D Pixels + New Technologies," Conference contribution to VERTEX 2009, September 13-19, 2009, Veluwe, The Netherlands

[9] H. Kagan et al., "Radiation Sensors for High Energy Physics Experiments," CVD Diamond for Electronic Devices and Sensors" 207-226, (2009), John Wiley \& Sons, Ltd 